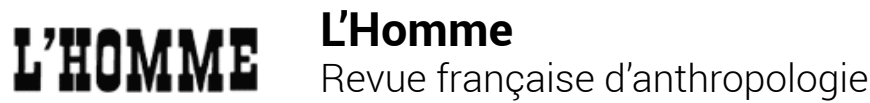

163 | juillet-sptembre 2002

De la légende au mythe. Parole, langue et pensée

Jean Michaud, s. dir., Turbulent Times and Enduring Peoples. Mountain Minorities in the South-East Asian Massif

Richmond, Curzon Press, 2000, XIII + 255 p., bibl., index, fig., tabl.

\section{Bernard Formoso}

\section{(2) OpenEdition}

\section{Journals}

Édition électronique

URL : http://journals.openedition.org/lhomme/12681

DOI : 10.4000//homme.12681

ISSN : 1953-8103

Éditeur

Éditions de l'EHESS

Édition imprimée

Date de publication : 21 juin 2002

Pagination : 290-292

ISBN : 2-7132-1771-7

ISSN : 0439-4216

\section{Référence électronique}

Bernard Formoso, "Jean Michaud, s. dir., Turbulent Times and Enduring Peoples. Mountain Minorities in the South-East Asian Massif », L'Homme [En ligne], 163 | juillet-sptembre 2002, mis en ligne le 10 juillet 2007, consulté le 24 septembre 2020. URL : http://journals.openedition.org//homme/12681 ; DOI : https://doi.org/10.4000//homme.12681

Ce document a été généré automatiquement le 24 septembre 2020

(C) École des hautes études en sciences sociales 


\title{
Jean Michaud, s. dir., Turbulent Times and Enduring Peoples. Mountain Minorities in the South-East Asian Massif
}

Richmond, Curzon Press, 2000, XIII + 255 p., bibl., index, fig., tabl.

\author{
Bernard Formoso
}

1 L'ORIGINALITÉ de cet ouvrage, soulignée par Jean Michaud et John McKinnon dans l'introduction, tient au fait qu'il est le premier à embrasser l'histoire et la situation contemporaine des Montagnards de plusieurs pays de la Péninsule indochinoise. En effet, les autres recueils de ce type, pour être nombreux depuis les années 1970, centrent leur attention soit sur les «tribaux » de Thaillande, soit sur les "minorités nationales» de la République Populaire de Chine. Or, font justement remarquer les coéditeurs du livre, on ne peut comprendre le destin de peuples qui ont toujours su se jouer des frontières si l'on n'adopte pas une perspective transnationale. Dépasser l'approche classique de l'ethnie, qualifiée selon les cas de substantialiste ou d'essentialiste, et qui objectifie la culture des groupes en une somme discrète de traits, est une autre finalité de l'ouvrage. On regrette néanmoins qu'à aucun moment de la synthèse introductive ne soient mentionnés les noms d'Edmund Leach et de Michael Moerman $^{1}$, qui, concernant l'étude de sociétés d'Asie du Sud-Est, furent pourtant les précurseurs d'un déplacement salutaire de perspective d'un supposé « cœur » réifié de l'ethnie vers les frontières socio-culturelles des groupes en contact.

2 Le recueil rassemble en tout dix contributions, introduction comprise, dont quatre consacrées aux Montagnards du nord de la Thaillande, deux à leurs homologues du Vietnam, deux à ceux de Birmanie et une à ceux du sud de la Chine. Les Hmong (branche de la famille Miao-Yao) et les Karen, deux groupes de populations parmi les plus étudiés de la Péninsule, sont une fois encore privilégiés, puisque six des neuf études de cas les ont pris pour objet. Par contre, les Austro-Asiatiques, ceux que l'on a parfois appelés Proto-Indochinois du fait de l'ancienneté de leur implantation dans la 
région, ne sont pas mieux pris en compte que dans les ouvrages analogues parus antérieurement.

Du point de vue thématique, certains des articles offrent malgré tout d'utiles éclairages sur des aspects jusqu'alors négligés de la vie des Karen et des Miao-Yao. Il en va ainsi de leur ethnohistoire, à comprendre non pas comme un recueil des modes émiques de représentation du passé, mais comme la compilation analytique des matériaux collectés à leur propos au fil du temps par des agents extérieurs. Dans cette optique, Jean Michaud conduit une très intéressante étude de l'ethnographie coloniale au Tonkin qui, outre l'examen approfondi des modes d'action plus ou moins cohérents de l'administration française à l'endroit des minorités, rend justice au rigoureux travail d'observation fourni par d'humbles officiers comme le lieutenant-colonel Bonifacy ou les capitaines Fesch et Reverony. Alison Lewis, pour sa part, analyse l'action des missionnaires protestants d'origine rurale, tels Samuel Clarke, W. S. Fleming ou James Adams, auprès des Hmong du Guizhou. Il montre que le pragmatisme paysan des pasteurs entrant bien vite en phase avec celui des locaux, cela eut pour effet la formation massive de prêtres hmong, y compris des femmes, qui leur offrit les clés d'une promotion sociale par la maitrise de l'écriture. Néanmoins, il ressort de l'étude que les chrétiens et leur logique manichéenne introduisirent des clivages sociaux supplémentaires dans une société déjà très segmentée.

Clive Christie, de son côté, retrace l'histoire moderne des Karen sur la base d'une périodisation originale qui distingue entre époques coloniale, anticoloniale et postcoloniale. Son analyse du processus qui conduisit à l'émergence d'un ethnonationalisme karen prend appui sur les écrits jusqu'alors négligés d'intellectuels autochtones comme San C. Po ou Saw Po Chit. Bien moins originale est l'évocation des migrations hmong entre la Chine et le Laos par Christian Culas. Celui-ci rouvre un dossier déjà bien documenté (cf. les travaux de Maurice Abadie, A. Raquez, Émile Lunet de Lajonquière, Joel Halpern, Jacques Lemoine, Robert Cooper et Nicholas Tapp), sans vraiment apporter de nouveaux éclairages sur le sujet. Enfin, François Robinne décrit l'évolution des rapports interethniques autour du lac Inle, au Myanmar, évolution marquée par le renversement des rapports de force entre les Shan et les Intha, ces derniers ayant pris le contrôle des institutions politiques et religieuses, sans que l'article en lui-même nous informe sur les causes d'une telle mutation. Celles-ci sont mieux explicitées dans l'ouvrage consacré à ces aspects que Robinne a récemment publié et dont la présente contribution est un résumé un peu terne ${ }^{2}$.

Parallèlement à l'ethnohistoire des minorités de la Péninsule, l'ouvrage aborde le problème de leur intégration aux ensembles nationaux contemporains et de l'attitude des États à leur endroit. Oscar Salemink propose ainsi une étude bien documentée sur la politique que le gouvernement vietnamien poursuit depuis la fin des années 1980 afin de sédentariser les minorités des hauts plateaux du centre du Vietnam et d'opérer une « préservation sélective » de leurs coutumes. L'objectif implicite des mesures prises dans ce cadre est bien sûr de vietnamiser sans heurts les Montagnards. Ces derniers résistent malgré tout et, explique Salemink, l'acte le plus manifeste en ce sens est leur conversion massive au christianisme. Peter Kunstadter présente une synthèse succincte d'une étude par questionnaires qu'il a conduite en 1987-1988 auprès de 198 villages thaïlandais où vivaient des Hmong. L'enquête portait sur des projets de développement conduits localement et leur impact. Elle confirme sur le plan statistique des tendances relevées par d'autres auteurs, comme le fait que ces projets soient de préférence mis en 
œuvre dans des localités proches des routes et des centres de marché, que, malgré tout, dans les deux tiers des cas une ou plusieurs des cultures commerciales soumises à expérimentation aboutissent à un échec, ou bien encore qu'il existe une corrélation forte au niveau villageois entre la présence de structures sanitaires et une forte baisse de la consommation d'opium.

6 L'extension du réseau routier thaïlandais aux régions peuplées de Montagnards a favorisé non seulement l'essaimage des projets de développement, mais aussi l'intrusion des touristes, via le trekking. Henry Bartsch examine les conséquences de cette activité sur un village karen. Il note que l'élévation du niveau de vie d'une minorité se trouve contrebalancée par les nouvelles dépendances que crée pour beaucoup de familles l'impossibilité de concilier agriculture et services touristiques. De plus, l'usage qui consiste à faire fumer de l'opium aux hôtes étrangers renforce l'accoutumance à la drogue de certains Karen. Enfin, dans le dernier chapitre, Hjorleifur Jonsson donne une interprétation textuelle, à la manière de Clifford Geertz, d'une foire associant plusieurs villages miao-yao, organisée à l'initiative d'enseignants, et qui avait pour temps forts un discours du député local, des compétitions sportives intervillageoises et des danses folkloriques. Autant de manifestations qui servent de prétextes à l'auteur pour poser la question du rapport des Hmong et des Mien à l'Étatnation thaillandais.

7 Finalement, comme c'est souvent le cas pour ce genre de recueil, l'ouvrage manque d'unité, tant les thèmes abordés au fil des contributions sont variés. L'idée suivant laquelle il faut adopter une perspective transnationale pour traiter des minorités de la Péninsule retient l'attention. Cependant, le concept de «massif sud-est asiatique » que Jean Michaud et John Mc Kinnon considèrent comme " un espace social signifiant » à la suite de Lim Joo-Jock ${ }^{3}$, pose problème. D'une part, parce qu'ils ne définissent jamais précisément les contours de cet espace montagneux; d'autre part, parce qu'à y regarder de plus près, une notion aussi englobante ne fait guère sens, aussi bien du point de vue géographique ou écologique (on a affaire à plusieurs chaînes montagneuses et à une multitude d'écosystèmes), que du point de vue des relations interethniques. En effet, l'hypothèse selon laquelle les Montagnards du nord et du sud de la Péninsule indochinoise partagent un seul et même espace social est d'une validité douteuse.

\section{NOTES}

1. Edmund Leach, Political Systems of Highland Burma, Boston, Beacon Press, 1954 ; Michael Moerman, « Ethnic Identification in a Complex Civilization : Who are the Lue ? ", American Anthropologist, 1965, 67 : 1215-1230.

2. François Robinne, Fils et maîtres du Lac, Paris, CNRS Éditions/Éditions de la MSH, 2000 («Chemins de l'ethnologie »). [Voir le compte rendu de ce livre par Emmanuel Guillon dans L'Homme, 2002, 161 : 283-286. Ndlr.] 
3. Lim Joo-Jock, Territorial Power Domains, South-East Asia and China. The Geo-Strategy of an Overarching Massif, Singapore, Institute of South-East Asian Studies, 1984.

\section{AUTEUR}

BERNARD FORMOSO

Université Paris X, Laboratoire d'ethnologie et de sociologie comparative, Nanterre. 